Lund-Mph-98/10

\title{
Zee Mass Matrix and Bi-Maximal Neutrino Mixing
}

\author{
Cecilia Jarlskog ⿵a $^{a}$, Masahisa Matsuda $\Pi^{a}$ \\ Solveig Skadhauge fa and Morimitsu Tanimoto $\mathrm{f}^{a}$ \\ a Department of Mathematical Physics, LTH, Lund University, S-22100, Lund, Sweden \\ ${ }^{b}$ Science Education Laboratory, Ehime University, 790-8577 Matsuyama, JAPAN
}

\begin{abstract}
We investigate neutrino masses and mixings within the framework of the Zee mass matrix, with three lepton flavors. It is shown that the bi-maximal solution is the only possibility to reconcile atmospheric and solar neutrino data, within this ansatz. We obtain two almost degenerate neutrinos, which are mixtures of all three neutrino flavors, with heavy masses $\simeq \sqrt{\Delta m_{\text {atm }}^{2}}$. The predicted mass of the lightest neutrino, which should consist mostly of $\nu_{\mu}$ and $\nu_{\tau}$, is $\simeq \Delta m_{\odot}^{2} /\left(2 \sqrt{\Delta m_{\text {atm }}^{2}}\right)$.

PACS: 14.60.Pq, 14.60.Lm, 12.60.Fr
\end{abstract}

*E-mail address: cecilia.jarlskog@matfys.lth.se

$\dagger$ Permanent address:Department of Physics and Astronomy, Aichi University of Education, Kariya, Aichi 448, Japan. E-mail address:mmatsuda@matfys.lth.se

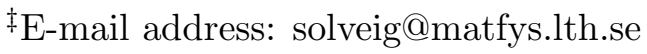

$\S$ E-mail address: tanimoto@edserv.ed.ehime-u.ac.jp 
In the past few years stronger experimental signals, than ever before, have been seen for neutrino oscillations. Recent atmospheric neutrino experiments [1,2] indicate oscillations among neutrino flavors with large mixing angles [3]. The simplest solution to the solar neutrino deficit problem, observed by the Super-Kamiokande experiment [4] as well as other experiments [5], is again neutrino oscillations. Indeed, the field of neutrino oscillations is expected to enter into a new era, with the start of long baseline (LBL) neutrino experiments [6] [ These experiments will hopefully solve the present neutrino anomalies.

Since the CHOOZ experiment [9] excludes oscillation of $\nu_{\mu} \rightarrow \nu_{e}$ with a large mixing angle for $\Delta m^{2} \geq 9 \times 10^{-4} \mathrm{eV}^{2}$, large mixing between $\nu_{\mu}$ and $\nu_{\tau}$ is the simplest interpretation of the atmospheric $\nu_{\mu}$ deficit. The difference between the quark-mixing and lepton-mixing matrices is striking. The Cabibbo-Kobayashi-Maskawa mixing matrix $V_{C K M}$ [10] in the quark sector is described by small mixing angles among different flavors but in the leptonic sector [11] at least one large mixing angle seems to be needed. It will be important to understand why these patterns are so different.

Another issue, to be understood, is why the neutrino masses are so small? The most popular answer to the latter question is given by the see-saw mechanism [12] which introduces heavy right-handed Majorana neutrinos with masses of the order $10^{10}-10^{16} \mathrm{GeV}$. This attractive model has been extensively studied in the literature. However, it is important to consider also other possible scenarios with small neutrino masses, specially extensions of the standard model (SM) at a low energy scale. The Zee model [13] is such an alternative and has been studied in the literatures for almost twenty years [14 18]. In this paper, we will discuss the present status of the Zee mass matrix, in the light of recent experimental results.

In the Zee model [13] neutrino masses are generated by radiative corrections, and hence the model may provide an explanation of the smallness of neutrino masses. In this model, the following Lagrangian is added to the SM;

$$
\begin{aligned}
\mathcal{L}= & \sum_{l, l^{\prime}=e, \mu, \tau} f_{l l^{\prime}} \overline{\Psi_{l L}} i \sigma_{2}\left(\Psi_{l^{\prime} L}\right)^{c} h^{-}+\mu \Phi_{1}^{T} i \sigma_{2} \Phi_{2} h^{-}+\text {h.c. } \\
= & 2 f_{e \mu}\left[\overline{\nu_{e L}}\left(\mu_{L}\right)^{c}-\bar{e}_{L}\left(\nu_{\mu L}\right)^{c}\right] h^{-}+2 f_{e \tau}\left[\overline{\nu_{e L}}\left(\tau_{L}\right)^{c}-\bar{e}_{L}\left(\nu_{\tau L}\right)^{c}\right] h^{-} \\
& +2 f_{\mu \tau}\left[\overline{\nu_{\mu L}}\left(\tau_{L}\right)^{c}-\bar{\mu}_{L}\left(\nu_{\tau L}\right)^{c}\right] h^{-}+\mu\left(\Phi_{1}^{+} \Phi_{2}^{0}-\Phi_{1}^{0} \Phi_{2}^{+}\right) h^{-}+h . c .
\end{aligned}
$$

where $\Psi_{l L}=\left(\nu_{l}, l\right)_{L}^{T}, \Phi_{i}=\left(\Phi_{i}^{+}, \Phi_{i}^{0}\right)^{T}, i=1,2$. The Higgs potential is omitted here. The charged Zee boson, $h^{ \pm}$, is a singlet under $S U(2)_{L}$. We need at least two Higgs doublets in order to make the Zee mechanism viable, since the antisymmetric coupling to the Zee boson is the cause of $B-L$ violation, and hence of Majorana masses. Note that only $\Phi_{1}$ couples to leptons, as in the SM. The mass matrix, generated by radiative correction at one loop level [13 18], is given by

$$
\left(\begin{array}{ccc}
0 & m_{e \mu} & m_{e \tau} \\
m_{e \mu} & 0 & m_{\mu \tau} \\
m_{e \tau} & m_{\mu \tau} & 0
\end{array}\right)
$$

where

$$
m_{e \mu}=f_{e \mu}\left(m_{\mu}^{2}-m_{e}^{2}\right) \frac{\mu v_{2}}{v_{1}} F\left(M_{1}^{2}, M_{2}^{2}\right)
$$




$$
\begin{aligned}
& m_{e \tau}=f_{e \tau}\left(m_{\tau}^{2}-m_{e}^{2}\right) \frac{\mu v_{2}}{v_{1}} F\left(M_{1}^{2}, M_{2}^{2}\right) \\
& m_{\mu \tau}=f_{\mu \tau}\left(m_{\tau}^{2}-m_{\mu}^{2}\right) \frac{\mu v_{2}}{v_{1}} F\left(M_{1}^{2}, M_{2}^{2}\right)
\end{aligned}
$$

and

$$
F\left(M_{1}^{2}, M_{2}^{2}\right)=\frac{1}{16 \pi^{2}} \frac{1}{M_{1}^{2}-M_{2}^{2}} \ln \frac{M_{1}^{2}}{M_{2}^{2}} .
$$

The parameter $v_{1(2)}$ is the vacuum expectation value of the neutral component of the Higgs doublet $\Phi_{1(2)}$. $M_{1}$ and $M_{2}$ are the masses of the physical particles defined by the fields

$$
H_{1}^{+}=h^{+} \cos \phi-\Phi^{+} \sin \phi \quad, \quad H_{2}^{+}=h^{+} \sin \phi+\Phi^{+} \cos \phi
$$

where $\Phi^{+}$is the charged Higgs boson that would have been a physical particle in the absence of the $h^{+}$. Finally, the mixing angle $\phi$ is defined by

$$
\tan 2 \phi=\frac{4 \sqrt{2} \mu M_{W}}{g \sqrt{\left(M_{1}^{2}-M_{2}^{2}\right)^{2}-\left(4 \sqrt{2} g^{-1} \mu M_{W}\right)^{2}}} .
$$

Due to the antisymmetry of the coupling matrix, $f_{l l^{\prime}}=-f_{l^{\prime} l}$, the Zee model requires all diagonal elements in Eq. (2) to vanish at one loop level. Small corrections will however be obtained at higher orders in perturbation theory. Hereafter, we refer to the above matrix, with vanishing diagonal elements, as the Zee mass matrix.

The parameters $m_{e \mu}, m_{e \tau}, m_{\mu \tau}$ in the Zee mass matrix are not described by the eigenvalues $m_{i}(i=1,2,3)$ due to the traceless property of the matrix, leaving only two independent observable parameters. In the same way it is impossible to represent the mixing matrix $U$ by using $m_{i}$. In literature [14]18], it has been assumed that there is a hierarchy such as $m_{e \mu} \ll m_{e \tau}, m_{\mu \tau}$ and the neutrino masses and mixings are discussed under such assumptions. This hierarchy is natural if the coupling constants $f_{l l^{\prime}}$ are of the same order of magnitude. However, we would like to explore all possibilities of masses and mixings in the Zee model by relaxing this assumption. Instead we use recent atmospheric neutrino data [1.2] as input to determine patterns in the Zee mass matrix that are viable.

The neutrino mass matrix $M_{\nu}$ is generally, for Majorana particles, constructed by

$$
M_{\nu}=U M^{\text {diag. }} \cdot U^{T}
$$

due to its symmetric property $M_{\nu}=M_{\nu}^{T}$. The mixing matrix $U$, called the MNS mixing matrix [11], is defined in the basis where the mass matrix of charged leptons is diagonal, with masses $m_{e, \mu, \tau}$. Furthermore,

$$
M^{\text {diag. }}=\left(\begin{array}{ccc}
m_{1} & 0 & 0 \\
0 & m_{2} & 0 \\
0 & 0 & m_{3}
\end{array}\right) .
$$


For Majorana neutrinos there are three phases in the matrix $U$ and this is generally given by

$$
U=\left(\begin{array}{ccc}
c_{1} c_{3} & s_{1} c_{3} e^{i \beta} & s_{3} e^{-i(\delta-\alpha)} \\
-s_{1} c_{2}-c_{1} s_{2} s_{3} e^{i \delta} & \left(c_{1} c_{2}-s_{1} s_{2} s_{3} e^{i \delta}\right) e^{i \beta} & s_{2} c_{3} e^{i \alpha} \\
s_{1} s_{2}-c_{1} c_{2} s_{3} e^{i \delta} & \left(-c_{1} s_{2}-s_{1} c_{2} s_{3} e^{i \delta}\right) e^{i \beta} & c_{2} c_{3} e^{i \alpha}
\end{array}\right)
$$

where $c_{i} \equiv \cos \theta_{i}$ and $s_{i} \equiv \sin \theta_{i}$. The Zee mass matrix exhibits no $C P$ violation [19] and we will therefore neglect the phases in our investigation. The diagonal elements in $M_{\nu}$ are given by

$$
\begin{aligned}
& (1,1) \quad m_{1} c_{1}^{2} c_{3}^{2}+m_{2} s_{1}^{2} c_{3}^{2}+m_{3} s_{3}^{2}, \\
& (2,2) m_{1}\left(s_{1} c_{2}+c_{1} s_{2} s_{3}\right)^{2}+m_{2}\left(c_{1} c_{2}-s_{1} s_{2} s_{3}\right)^{2}+m_{3} s_{2}^{2} c_{3}^{2}, \\
& (3,3) \quad m_{1}\left(s_{1} s_{2}-c_{1} c_{2} s_{3}\right)^{2}+m_{2}\left(c_{1} s_{2}+s_{1} c_{2} s_{3}\right)^{2}+m_{3} c_{2}^{2} c_{3}^{2} .
\end{aligned}
$$

These should be zero (in general small) in the Zee model and we arrive at the following relations:

$$
m_{2}=-\frac{\cos ^{2} \theta_{1}-\tan ^{2} \theta_{3}}{\sin ^{2} \theta_{1}-\tan ^{2} \theta_{3}} m_{1}, \quad m_{3}=-m_{1}-m_{2}
$$

The second equality is obvious by the traceless property of the Zee mass matrix. Two of three masses have the same sign and the remaining one has opposite sign, which implies that one of the fields has opposite CP parity as compared to the other two. The dependence of mass eigenvalues on $\theta_{1}$ and $\theta_{3}$ is shown in Fig.1. Inserting (11) into (10) gives the relation

$$
\cos 2 \theta_{1} \cos 2 \theta_{2} \cos 2 \theta_{3}=\frac{1}{2} \sin 2 \theta_{1} \sin 2 \theta_{2}\left(3 \cos ^{2} \theta_{3}-2\right) \sin \theta_{3} .
$$

This equation means that the three mixing angles are not independent. For typical values of $\theta_{1}, \theta_{2}, \theta_{3}$ the structure of the mixing matrix is discussed later, using this equation.

Before entering into the analysis of the Zee mass matrix, we give a short survey of recent neutrino experiments. Our approach is to assume that oscillations account for the solar and atmospheric neutrino data, thus pinning down two mass squared differences, which is the maximal number of mass differences in the model we are investigating. If the results of LSND [20] would be confirmed by KARMEN 21] or any other experiment, the model examined in this paper would no longer be relevant. The deficit of $\nu_{\mu}$ in recent Super Kamiokande data [1], is interpreted as oscillation of $\nu_{\mu} \rightarrow \nu_{\tau}$ with nearly maximal mixing angle in a two flavor analysis [3,22]. These results yield

$$
\Delta m_{a t m}^{2} \simeq(0.5-6) \times 10^{-3} \mathrm{eV}^{2}, \quad \sin ^{2} 2 \theta_{a t m}>0.82 \quad(90 \% \text { C.L }) .
$$

Further, the deficit in the solar neutrino experiments suggests the following best-fit solutions [23] as

(1) MSW small angle solution; $\Delta m_{\odot}^{2} \simeq 5.4 \times 10^{-6} \mathrm{eV}^{2}, \sin ^{2} 2 \theta_{\odot} \simeq 6 \times 10^{-3}$, 


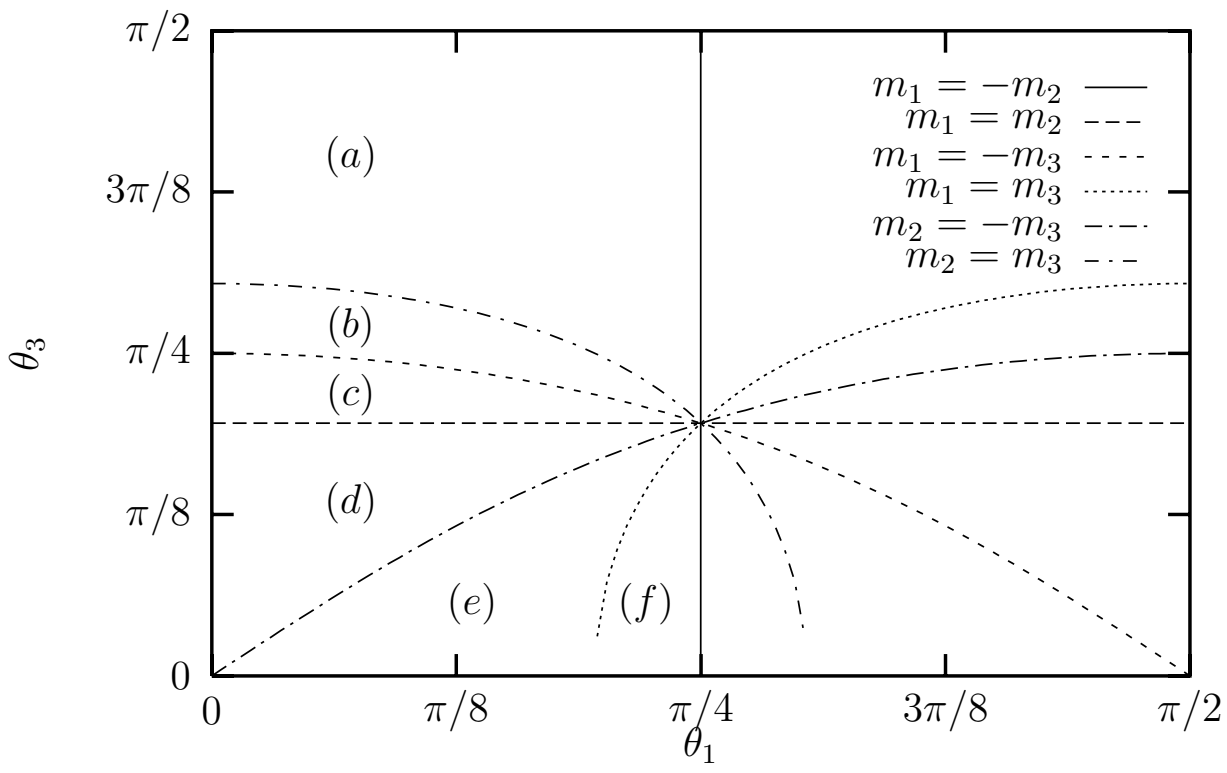

FIG. 1. The dependence of mass eigenvalues on the mixing angles $\theta_{1}$ and $\theta_{3}$. The lines represents special relations among the three masses as denoted in the figure. In the right hand side the domains are running oppositely, starting with (a) in the bottom and ending with (f) in the top. In region (a) we have $m_{1}>-m_{2}>-m_{3}$, (b) $m_{1}>-m_{3}>-m_{2}$, (c) $-m_{3}>m_{1}>m_{2}$, (d) $-m_{3}>m_{2}>m_{1}$, (e) $-m_{2}>m_{3}>m_{1}$, and (f) $-m_{2}>m_{1}>m_{3}$. Here the sign of $m_{1}$ is taken to be positive.

(2) MSW large angle solution; $\Delta m_{\odot}^{2} \simeq 1.8 \times 10^{-5} \mathrm{eV}^{2}, \sin ^{2} 2 \theta_{\odot} \simeq 0.76$,

(3) "just-so" vacuum solution; $\Delta m_{\odot}^{2} \simeq 6.5 \times 10^{-11} \mathrm{eV}^{2}, \sin ^{2} 2 \theta_{\odot} \simeq 0.75$.

It is noted that the large angle MSW solution seems to be excluded by the simultaneous fits to all the available data [23]. As there are still theoretical uncertainties about this [24, we will keep this possibility in our considerations. The combined results of atmospheric and solar neutrino experiments suggest that there exist two hierarchical mass squared differences $\Delta m_{a t m}^{2} \gg \Delta m_{\odot}^{2}$. Further, the component $U_{e 3}$ in Eq.(9) should be small, as suggested by the CHOOZ experiment [9]. The value $\sin ^{2} 2 \theta_{C H O O Z}<0.18 \mathrm{im}$ plies $\left|U_{e 3}\right|<0.22$ for $\Delta m^{2} \geq 9 \times 10^{-4} \mathrm{eV}^{2}$ [25]. For the MSW small angle solution, case (1), together with the atmospheric results, a possible mixing matrix has the form

$$
U_{1} \simeq\left(\begin{array}{ccc}
1 & \epsilon_{1} & \epsilon_{2} \\
\epsilon_{3} & c & s \\
\epsilon_{4} & -s & c
\end{array}\right)
$$

where $c \simeq s \simeq 1 / \sqrt{2}$. This mixing matrix might be realized in the case;

$$
\left|\Delta m_{32}^{2}\right| \simeq\left|\Delta m_{31}^{2}\right| \simeq \Delta m_{a t m}^{2}, \quad\left|\Delta m_{21}^{2}\right| \simeq \Delta m_{\odot}^{2}
$$

which demands $m_{3} \gg m_{i} \geq m_{j}$ or $m_{i} \geq m_{j} \gg m_{3}(i, j=1,2$ or 2,1$)$ or 


$$
\left|\Delta m_{32}^{2}\right| \simeq\left|\Delta m_{21}^{2}\right| \simeq \Delta m_{a t m}^{2}, \quad\left|\Delta m_{31}^{2}\right| \simeq \Delta m_{\odot}^{2}
$$

implying $m_{2} \gg m_{i} \geq m_{j}$ or $m_{i} \geq m_{j} \gg m_{2}(i, j=1,3$ or 3,1$)$. Another solution for MSW small angle mixing is

$$
U_{1}^{\prime} \simeq\left(\begin{array}{ccc}
\epsilon_{1} & 1 & \epsilon_{2} \\
c & \epsilon_{3} & s \\
-s & \epsilon_{4} & c
\end{array}\right)
$$

This type of mixing suggests

$$
\left|\Delta m_{32}^{2}\right| \simeq\left|\Delta m_{31}^{2}\right| \simeq \Delta m_{a t m}^{2}, \quad\left|\Delta m_{21}^{2}\right| \simeq \Delta m_{\odot}^{2}
$$

or

$$
\left|\Delta m_{31}^{2}\right| \simeq\left|\Delta m_{21}^{2}\right| \simeq \Delta m_{a t m}^{2}, \quad\left|\Delta m_{32}^{2}\right| \simeq \Delta m_{\odot}^{2}
$$

and we obtain the solution $m_{1} \gg m_{i} \geq m_{j}$ or $m_{i} \geq m_{j} \gg m_{1}(i, j=2,3$ or 3,2$)$ for the latter case.

For the "just-so" and MSW large angle solutions a typical mixing matrix is

$$
\left(\begin{array}{ccc}
c & s & \epsilon_{1} \\
-s & c & \epsilon_{2} \\
\epsilon_{3} & \epsilon_{4} & 1
\end{array}\right) \quad \text { or } \quad\left(\begin{array}{ccc}
c & s & \epsilon_{1} \\
\epsilon_{2} & \epsilon_{3} & 1 \\
-s & c & \epsilon_{4}
\end{array}\right)
$$

Neither of these is compatible with the maximal mixing pattern of the atmospheric $\nu_{\mu} \rightarrow$ $\nu_{\tau}$ oscillation, and must be discarded for this reason. We are left with one possibility;

$$
U_{3} \simeq\left(\begin{array}{ccc}
c^{\prime} & s^{\prime} & \epsilon \\
-c s^{\prime} & c c^{\prime} & s \\
s s^{\prime} & -s c^{\prime} & c
\end{array}\right)
$$

for interpreting large angle solar neutrino solutions, where $c \simeq s \simeq c^{\prime} \simeq s^{\prime} \simeq 1 / \sqrt{2}$. In the limit $\epsilon=0$ this is known as the bi-maximal mixing matrix [26]. Nearly bi-maximal mixing is discussed in Ref. [27]. Taking

$$
\left|\Delta m_{32}^{2}\right| \simeq\left|\Delta m_{31}^{2}\right| \simeq \Delta m_{a t m}^{2}, \quad\left|\Delta m_{21}^{2}\right| \simeq \Delta m_{\odot}^{2}
$$

with $m_{3} \gg m_{i} \geq m_{j}$ or $m_{i} \geq m_{j} \gg m_{3}, \quad(i, j=1,2$ or 2,1$)$, it has been shown that the mixing matrix $U_{3}$ is consistent with vacuum solution for both solar and atmospheric neutrino anomalies within the experimental uncertainties [28]. The MSW large angle solution could also be accommodated here, but then the allowed parameter space is rather small.

Furthermore the degenerate case $m_{1} \simeq m_{2} \simeq m_{3}$ is another possibility for all the above cases. Using the definition;

$$
m_{a}=m_{0}, \quad m_{b}=m_{0}\left(1-\frac{\delta}{2}\right), \quad m_{c}=m_{0}\left(1-\frac{\delta+\epsilon}{2}\right),
$$


we can assign $a, b, c$ to each of 1,2,3 according to the mass relation obtained above. Only in this degenerate case is it possible to have a mass $m_{0}=\mathcal{O}(1) \mathrm{eV}$ for the neutrinos, as is suggested by the hot dark matter argument. Setting $m_{0}=1 \mathrm{eV}$ the parameters $\delta$ and $\epsilon$ take the values $\mathcal{O}\left(10^{-3}-10^{-2}\right)$ and $\mathcal{O}\left(10^{-5}\right)$ respectively to reproduce $\Delta m_{a b}^{2} \simeq \Delta m_{a c}^{2} \simeq \Delta m_{a t m}^{2}$ and $\Delta m_{b c}^{2} \simeq \Delta m_{\odot}^{2}$.

We now return to the analysis of the Zee mass matrix. In accordance with the data of atmospheric neutrino experiments we require $\theta_{2} \simeq \pi / 4$. Now Eq.(12) implies that we have the following possibilities; $\theta_{3} \simeq 0$ or $\theta_{1} \simeq 0$ or $\theta_{3} \simeq \arctan \sqrt{1 / 2}$. The latter case will be commented on later. We will only consider the case of $\theta_{1} \simeq 0$ together with $\theta_{3} \simeq 0$, due to the experimental constraints on $U_{e 3}$. To begin with we concentrate on the solution $\theta_{3} \simeq 0$.

If we take the extreme limit $\theta_{2}=\pi / 4$ and $\theta_{3}=0$, the mixing matrix becomes

$$
U_{a t m} \simeq\left(\begin{array}{ccc}
c_{1} & s_{1} & 0 \\
-\frac{s_{1}}{\sqrt{2}} & \frac{c_{1}}{\sqrt{2}} & \frac{1}{\sqrt{2}} \\
\frac{s_{1}}{\sqrt{2}} & -\frac{c_{1}}{\sqrt{2}} & \frac{1}{\sqrt{2}}
\end{array}\right)
$$

It is noted that the extreme case does not change the qualitative structure of the model and is consistent with combined SK and CHOOZ data in a three flavor analyses [3,22]. In this limit we obtain the constraints

$$
m_{1} c_{1}^{2}+m_{2} s_{1}^{2}=0, \quad m_{1} s_{1}^{2}+m_{2} c_{1}^{2}+m_{3}=0
$$

from Eq.(10). Then the parameters satisfy the constraint

$$
\tan ^{2} \theta_{1}=-\frac{m_{1}}{m_{2}}>0
$$

and we arrive at

$$
\begin{aligned}
M_{\nu} & =\left(\begin{array}{ccc}
0 & -\frac{c_{1} s_{1}}{\sqrt{2}}\left(m_{1}-m_{2}\right) & \frac{c_{1} s_{1}}{\sqrt{2}}\left(m_{1}-m_{2}\right) \\
-\frac{c_{1} s_{1}}{\sqrt{2}}\left(m_{1}-m_{2}\right) & 0 & -\frac{1}{2}\left(m_{1} s_{1}^{2}+m_{2} c_{1}^{2}-m_{3}\right) \\
\frac{c_{1} s_{1}}{\sqrt{2}}\left(m_{1}-m_{2}\right) & -\frac{1}{2}\left(m_{1} s_{1}^{2}+m_{2} c_{1}^{2}-m_{3}\right) & 0
\end{array}\right) \\
& =\left(\begin{array}{ccc}
0 & \pm \sqrt{\frac{\left|m_{1} m_{2}\right|}{2}} & \mp \sqrt{\frac{\left|m_{1} m_{2}\right|}{2}} \\
\pm \sqrt{\frac{\left|m_{1} m_{2}\right|}{2}} & 0 & -m_{1}-m_{2} \\
\mp \sqrt{\frac{\left|m_{1} m_{2}\right|}{2}} & -m_{1}-m_{2} & 0
\end{array}\right),
\end{aligned}
$$

where the upper (lower) sign corresponds to the case $m_{1}<0\left(m_{1}>0\right)$. The mixing matrix becomes

$$
U=\left(\begin{array}{ccc}
\sqrt{\frac{\left|m_{2}\right|}{\left|m_{1}\right|+\left|m_{2}\right|}} & \sqrt{\frac{\left|m_{1}\right|}{\left|m_{1}\right|+\left|m_{2}\right|}} & 0 \\
-\sqrt{\frac{\left|m_{1}\right|}{2\left(\left|m_{1}\right|+\left|m_{2}\right|\right)}} & \sqrt{\frac{\left|m_{2}\right|}{2\left(\left|m_{1}\right|+\left|m_{2}\right|\right)}} & \frac{1}{\sqrt{2}} \\
\sqrt{\frac{\left|m_{1}\right|}{2\left(\left|m_{1}\right|+\left|m_{2}\right|\right)}} & -\sqrt{\frac{\left|m_{2}\right|}{2\left(\left|m_{1}\right|+\left|m_{2}\right|\right)}} & \frac{1}{\sqrt{2}}
\end{array}\right) .
$$


As the two mass squared differences are hierarchical we have to stay close to the lines in Fig.(1). Due to the symmetry we only have to survey the left part of the parameter region, i.e $\theta_{1} \in[0, \pi / 4]$. Changing from the left to right side merely corresponds to an interchange of $m_{1} \leftrightarrow m_{2}$ and has no physical effect. We now consider the three possible values for $\theta_{1}$.

(I). The case with $\theta_{1} \simeq 0$. The Zee mass matrix then requires the following mass relations to hold;

$$
\left|m_{2}\right| \simeq\left|m_{3}\right| \gg\left|m_{1}\right| \quad, \quad \Delta m_{21}^{2} \simeq \Delta m_{31}^{2}=\Delta m_{a t m}^{2}, \quad \Delta m_{23}^{2}=\Delta m_{\odot}^{2} .
$$

In this case we get the mixing matrix;

$$
U_{1}^{Z e e}=\left(\begin{array}{ccc}
1 & \epsilon_{1} & 0 \\
-\frac{\epsilon_{1}}{\sqrt{2}} & \frac{1}{\sqrt{2}} & \frac{1}{\sqrt{2}} \\
\frac{\epsilon_{1}}{\sqrt{2}} & -\frac{1}{\sqrt{2}} & \frac{1}{\sqrt{2}}
\end{array}\right),
$$

with $\epsilon_{1} \simeq \sqrt{\left|m_{1} / m_{2}\right|}$. This corresponds to the small angle MSW solution $U_{1}$ (Eq.(14)). Nevertheless due to the mass relations in Eq.(29) the predicted probability of $\nu_{\mu} \rightarrow \nu_{\tau}$ oscillation,

$$
\begin{aligned}
P\left(\nu_{\mu} \rightarrow \nu_{\tau}\right) & =-4 \sum_{i>j} U_{\mu i} U_{\tau j}^{*} U_{\mu j}^{*} U_{\tau i} \sin ^{2} \frac{\Delta m_{i j}^{2} L}{4 E} \\
& \simeq 4 U_{\mu 1}^{2} U_{\tau 1}^{2} \sin ^{2} \frac{\Delta m_{a t m}^{2} L}{4 E} \\
& =\epsilon_{1}^{4} \sin ^{2} \frac{\Delta m_{a t m}^{2} L}{4 E},
\end{aligned}
$$

is tiny in contradiction with the SK experiment. Here and in the following we neglect terms with $\Delta m_{\odot}^{2}$ in $P\left(\nu_{\mu} \rightarrow \nu_{\tau}\right)$. Thus, the Zee mass matrix and large angle solution are not compatible, for $\theta_{1} \simeq 0$. Setting $\theta_{1} \simeq \pi / 2$ would correspond to $m_{1} \leftrightarrow m_{2}$ compared to the current case. It also causes an interchange of the two first columns in $U_{1}^{Z e e}$, which yields the matrix in Eq.(17). However this must be discarded for the same reason. (II). Here we take $\theta_{1} \simeq \arctan 1 / \sqrt{2}$ and obtain

$$
\left|m_{1}\right| \simeq\left|m_{3}\right| \gg\left|m_{2}\right| \quad, \quad \Delta m_{12}^{2} \simeq \Delta m_{32}^{2}=\Delta m_{a t m}^{2}, \quad \Delta m_{13}^{2}=\Delta m_{\odot}^{2} .
$$

This case requires the mixing matrix to be

$$
U_{2}^{Z e e}=\left(\begin{array}{ccc}
\sqrt{\frac{2}{3}} & \sqrt{\frac{1}{3}} & 0 \\
-\sqrt{\frac{1}{6}} & \sqrt{\frac{1}{3}} & \sqrt{\frac{1}{2}} \\
\sqrt{\frac{1}{6}} & -\sqrt{\frac{1}{3}} & \sqrt{\frac{1}{2}}
\end{array}\right) .
$$

Giving the angles:

$$
\sin ^{2} 2 \theta_{\mathrm{CHOOZ}}=4 U_{e 2}^{2}\left(1-U_{e 2}^{2}\right) \simeq \frac{8}{9}, \quad \sin ^{2} 2 \theta_{a t m}=4 U_{\mu 2}^{2} U_{\tau 2}^{2} \simeq \frac{4}{9} .
$$


Hence this is also incompatible with experiments.

(III). In this third case $\theta_{1} \simeq \pi / 4$, whereby

$$
\left|m_{1}\right| \simeq\left|m_{2}\right| \gg\left|m_{3}\right| \quad, \Delta m_{13}^{2} \simeq \Delta m_{23}^{2}=\Delta m_{a t m}^{2}, \quad \Delta m_{12}^{2}=\Delta m_{\odot}^{2} .
$$

The mixing matrix is

$$
U_{3}^{Z e e}=\left(\begin{array}{ccc}
\frac{1}{\sqrt{2}} & \frac{1}{\sqrt{2}} & 0 \\
-\frac{1}{2} & \frac{1}{2} & \frac{1}{\sqrt{2}} \\
\frac{1}{2} & -\frac{1}{2} & \frac{1}{\sqrt{2}}
\end{array}\right)
$$

This corresponds to the solution given by $U_{3}$ in Eq.(21). The oscillation probability reads

$$
P\left(\nu_{\mu} \rightarrow \nu_{\tau}\right) \simeq \sin ^{2} \frac{\Delta m_{a t m}^{2} L}{4 E},
$$

in good agreement with experiments. Therefore we have a unique solution compatible with large angle in $\nu_{\mu} \rightarrow \nu_{\tau}$ within the ansatz of the Zee mass matrix. Due to the traceless property this implies

$$
\left|m_{1}\right| \simeq\left|m_{2}\right| \simeq \sqrt{\Delta m_{a t m}^{2}}, \quad\left|m_{3}\right| \simeq \frac{\Delta m_{\odot}^{2}}{2 \sqrt{\Delta m_{a t m}^{2}}},
$$

This is the case of "pseudo-Dirac" since $m_{1} \simeq-m_{2}$. The probabilities for other oscillation processes are as follows:

$$
\begin{aligned}
& P\left(\nu_{e} \rightarrow \nu_{e}\right) \simeq 1-\sin ^{2} \frac{\Delta m_{\odot}^{2} L}{4 E} \\
& P\left(\nu_{e} \rightarrow \nu_{\mu}\right) \simeq P\left(\nu_{e} \rightarrow \nu_{\tau}\right) \simeq \frac{1}{2} \sin ^{2} \frac{\Delta m_{\odot}^{2} L}{4 E}
\end{aligned}
$$

Thus the solar neutrinos are converted into an equal amounts of $\nu_{\mu}$ and $\nu_{\tau}$.

The mixing patterns $U_{1}^{Z e e}$ corresponds to the small angle MSW solution, and the matrix $U_{3}^{Z e e}$ corresponds to the large angle MSW or "just-so" solution. It is interesting to notice that the solution of three degenerate masses with $\mathcal{O}(1) \mathrm{eV}$ is not allowed due to the relation $m_{3}=-m_{1}-m_{2}$ and Eq.(26). This model requires naturally a hierarchical structure for mass matrix in the case of $\theta_{2}=\pi / 4$ and $\theta_{3}=0$. In conclusion only the bi-maximal solution, given in Eq.(36), is feasible, within the framework of the Zee mass matrix, whereas the solutions like $U_{1}^{Z e e}$ and $U_{2}^{Z e e}$ are not allowed.

Above, we have analyzed the Zee mass matrix at the limit $\theta_{3}=0$. It is also important to discuss the case of nonzero $\theta_{3}$ in order to obtain three flavor angles and to have predictions for future experiments. We parameterize this as

$$
\theta_{1}=\frac{\pi}{4}+\delta_{1}, \quad \theta_{2}=\frac{\pi}{4}+\delta_{2}, \quad \theta_{3}=\delta_{3}
$$

with $\delta_{i}(i=1,2,3) \ll 1$ and we will neglect terms of order $\delta_{i}^{2}$. The traceless property of the Zee mass matrix requires the relation $\delta_{3} \simeq 8 \delta_{1} \delta_{2}$, by using Eq.(12), and yields the approximate mixing matrix 


$$
U_{Z e e} \simeq\left(\begin{array}{ccc}
\frac{1-\delta_{1}}{\sqrt{2}} c_{3} & \frac{1+\delta_{1}}{\sqrt{2}} c_{3} & \delta_{3} \\
-\frac{1+\delta_{1}-\delta_{2}+\delta_{3}}{2} & \frac{1-\delta_{1}-\delta_{2}-\delta_{3}}{2} & \frac{1+\delta_{2}}{\sqrt{2}} c_{3} \\
\frac{1+\delta_{1}+\delta_{2}-\delta_{3}}{2} & -\frac{1-\delta_{1}+\delta_{2}+\delta_{3}}{2} & \frac{1-\delta_{2}}{\sqrt{2}} c_{3}
\end{array}\right)
$$

where $c_{3}=\sqrt{1-\delta_{3}^{2}}$ and the mass matrix is described as

$$
M_{\nu} \simeq\left(\begin{array}{ccc}
0 & -\frac{1-2 \delta_{1}-\delta_{2}}{\sqrt{2}} & \frac{1-2 \delta_{1}+\delta_{2}}{\sqrt{2}} \\
-\frac{1-2 \delta_{1}-\delta_{2}}{\sqrt{2}} & 0 & -4 \delta_{1} \\
\frac{1-2 \delta_{1}+\delta_{2}}{\sqrt{2}} & -4 \delta_{1} & 0
\end{array}\right) m_{1} .
$$

The ratio of lightest mass to heavy mass is approximately given by $\delta_{1}$ as shown in Eqs. 27, 42). Which restrictions do the present experiments give for the parameters $\delta_{1}, \delta_{2}, \delta_{3}$ ? Taking Eq.(13) and the lower limit for large angle solution of solar neutrino as $\sin ^{2} 2 \theta_{\odot}>0.65$ we obtain the following constraints;

$$
\begin{aligned}
\sin ^{2} 2 \theta_{a t m} & \simeq 4\left|U_{\mu 3}\right|^{2}\left|U_{\tau 3}\right|^{2} \simeq \frac{\left(1-2 \delta_{2}^{2}\right)\left(1-2 \delta_{3}^{2}\right)}{2} \geq 0.82, \\
\sin ^{2} 2 \theta_{\odot} & \simeq 4\left|U_{e 1}\right|^{2}\left|U_{e 2}\right|^{2} \simeq \frac{\left(1-2 \delta_{1}^{2}\right)\left(1-2 \delta_{3}^{2}\right)}{2} \geq 0.65 .
\end{aligned}
$$

These inequalities with $\left|\delta_{3}\right| \simeq 8\left|\delta_{1} \delta_{2}\right|$ leads to

$$
\delta_{1(2)}^{2} \leq \frac{1}{128 \delta_{2(1)}^{2}}\left(1-\frac{0.82(0.65)}{1-2 \delta_{2(1)}^{2}}\right) .
$$

We obtain the upper limit for $\delta_{3}$ to be

$$
\left|\delta_{3}\right| \leq 0.28
$$

If the data will be improved as $\sin ^{2} 2 \theta_{\text {atm }}>0.95, \sin ^{2} 2 \theta_{\odot}>0.95$ we get the upper limit $\left|\delta_{3}\right|<0.1$. A better estimate can nevertheless be deduced by noticing the following. The eigenvalues of $M_{\nu}$ are $m_{1},-\left(1-4 \delta_{1}\right) m_{1}, 4 \delta_{1} m_{1}$. In order to adjust the mass squared difference of $\Delta m_{\odot}^{2}$ using $\mathrm{Eq}\left([38), \delta_{1}\right.$ should be chosen as $\mathcal{O}\left(10^{-3}\right)$ for MSW or $\mathcal{O}\left(10^{-8}\right)$ for "just-so". This demands also $\delta_{3}$ to be tiny due to the relation $\delta_{3} \simeq 8 \delta_{1} \delta_{2}$. The value $\delta_{3} \simeq \mathcal{O}\left(10^{-3}\right)$ or $\mathcal{O}\left(10^{-8}\right)$ is well within the present experimental upper limit. In this case we expect no $\nu_{\mu} \rightarrow \nu_{e}$ oscillations at the LBL experiments [6 8] since,

$$
P\left(\nu_{\mu} \rightarrow \nu_{e}\right) \simeq 2 \delta_{3}^{2} \sin ^{2} \frac{\Delta m_{a t m}^{2} L}{4 E} \simeq 0
$$

We now briefly discuss the remaining solution: $\theta_{3}=\arctan (1 / \sqrt{2})+\delta_{3}, m_{1} \simeq m_{2} \simeq$ $-m_{3} / 2$, when requiring $\theta_{2}=\pi / 4+\delta_{2}$ in the Zee mass matrix. Here we take $\theta_{1}=\pi / 4+\delta_{1}$ to obtain the "maximal" case [29]. The mixing matrix reads

$$
U \simeq\left(\begin{array}{ccc}
\frac{1-\delta_{1}}{\sqrt{3}} & \frac{1+\delta_{1}}{\sqrt{3}} & \frac{1}{\sqrt{3}} \\
-\frac{1+\delta_{1}-\delta_{2}}{2}-\frac{1-\delta_{1}+\delta_{2}}{2 \sqrt{3}} & \frac{1-\delta_{1}-\delta_{2}}{2}-\frac{1+\delta_{1}+\delta_{2}}{2 \sqrt{3}} & \frac{1+\delta_{2}}{\sqrt{3}} \\
\frac{1+\delta_{1}+\delta_{2}}{2}-\frac{1-\delta_{1}-\delta_{2}}{2 \sqrt{3}} & -\frac{1-\delta_{1}+\delta_{2}}{2}-\frac{1+\delta_{1}-\delta_{2}}{2 \sqrt{3}} & \frac{1-\delta_{2}}{\sqrt{3}}
\end{array}\right)
$$


and

$$
M_{\nu} \simeq-\left(\begin{array}{ccc}
0 & 1-(2 \sqrt{3}-1) \delta_{2} & 1-(2 \sqrt{3}+1) \delta_{2} \\
1-(2 \sqrt{3}-1) \delta_{2} & 0 & 1-2 \sqrt{3} \delta_{2} \\
1-(2 \sqrt{3}+1) \delta_{2} & 1-2 \sqrt{3} \delta_{2} & 0
\end{array}\right) m_{1}
$$

Here $\delta_{3} \simeq-2 \sqrt{2} \delta_{1} \delta_{2} / \sqrt{3}$, from Eq.(12), and therefore terms with $\delta_{3}$ are neglected in the above matrices. This "maximal" case is only allowed for $\Delta m^{2}<9 \times 10^{-4} \mathrm{eV}^{2}$ due to results of the CHOOZ experiment [9]. However, a three flavor analysis [22] without CHOOZ data shows that the "maximal" solution lies in the region

$$
1.5 \times 10^{-3} \leq \Delta m_{32}^{2} \simeq \Delta m_{31}^{2} \simeq 3 m_{1}^{2} \leq 6.5 \times 10^{-3} \mathrm{eV}^{2}
$$

at 90\%C.L. Combining results from CHOOZ and SK experiments tends to exclude this "maximal" solution, which predicts large effects for $P\left(\nu_{\mu} \rightarrow \nu_{e}\right)$ in LBL experiments.

A comment on neutrino-less double beta decay is now in order. Although we get the heaviest mass $\left|m_{1}\right| \simeq\left|m_{2}\right| \simeq 0.02 \sim 0.08 \mathrm{eV}$ for bi-maximal mixing, neutrino-less double beta decay is forbidden in the Zee model. The effective neutrino mass in the decay

$$
\left\langle m_{\nu}\right\rangle=\left|\sum_{i} U_{e i}^{2} m_{\nu i}\right|
$$

is exactly zero due to the condition that $(1,1)$ entry in the mass matrix is zero. Generally, a theory which has zero entry in $(1,1)$ leads to vanishing neutrino-less double beta decay, even if the neutrino is a Majorana particle [30].

Returning to the Zee model which motivated the use of the Zee mass matrix, we find that the requirement of nearly bi-maximal mixing necessarily yields

$$
M_{\nu} \simeq\left(\begin{array}{ccc}
\delta_{1} & -1 & 1 \\
-1 & \delta_{1} & \delta_{1} \\
1 & \delta_{1} & \delta_{1}
\end{array}\right)
$$

By using the relation $m_{e \mu} \simeq m_{e \tau}$, in Eq.(Z), we obtain

$$
\frac{f_{e \mu}}{f_{e \tau}} \simeq \frac{m_{\tau}^{2}}{m_{\mu}^{2}} \simeq 2.9 \times 10^{2}
$$

and

$$
\frac{m_{e \tau}}{m_{\mu \tau}}=\frac{f_{e \tau}}{f_{\mu \tau}} \simeq \frac{\sqrt{2} \Delta m_{a t m}^{2}}{\Delta m_{\odot}^{2}} \simeq 10^{2} \text { or } 10^{7}
$$

for $\Delta m_{\text {atm }}^{2} \simeq 10^{-3} \mathrm{eV}^{2}$ and $\Delta m_{\odot}^{2} \simeq 10^{-5}$ or $10^{-10} \mathrm{eV}^{2}$. The magnitude of $f_{e \mu}$ may be estimated using Eq.(3) and $0.02<m_{e \mu}<0.08 \mathrm{eV}$

$$
f_{e \mu} \simeq(2 \sim 7)\left(\frac{100 \mathrm{GeV}}{\mu} \frac{1}{\tan \beta}\right) \times 10^{-4}
$$


where $\tan \beta=v_{2} / v_{1}$ and we have taken $M_{1}=200 \mathrm{GeV}$ and $M_{2}=300 \mathrm{GeV}$. We see that the relation of couplings must be of the form

$$
f_{e \mu} \gg f_{e \tau} \gg f_{\mu \tau}
$$

in order to agree with experiments. This indicates that most likely an extension of the Zee model is needed to give the anti-hierarchy above. This might be realized by assigning an approximate conserved $U(1)$ [31] charge

$$
L \equiv L_{e}-L_{\mu}-L_{\tau}
$$

which will strongly suppress $f_{\mu \tau}$. Although this cannot explain the factor of $\sim 10^{2}$ between $f_{e \mu}$ and $f_{e \tau}$, it can account for the more demanding factor of $10^{7}$ in the "just-so" case. With this extension the matrix in Eq.(51) can be derived from the Zee model. The tiny values on the diagonal will be caused by higher order effects, as mentioned before. Also it is remarkable that this will give an inverse mass hierarchy as $\left|m_{3}\right| \ll\left|m_{1}\right| \simeq\left|m_{2}\right|$. This pattern is substantially different from those studied in the previous analyses of the Zee model [18,32]. In Ref. [18], the case $\left|m_{1}\right| \ll\left|m_{2}\right| \simeq\left|m_{3}\right|$ is taken and the heavy masses $\left|m_{2}\right| \simeq\left|m_{3}\right|$ adjusted to be $(1-5) \mathrm{eV}$ as the candidates of hot dark matter and the mass squared differences are assigned to be $\Delta m_{32}^{2}=\Delta m_{a t m}^{2}, \Delta m_{31}^{2} \simeq \Delta m_{31}^{2} \simeq \Delta m_{L S N D}^{2}$. The addition of one sterile neutrino to the original Zee model is studied in Ref. [32], giving a possible explanation of all positive neutrino oscillation experiments, still keeping the assumption of $\left|f_{e \mu}\right| \simeq\left|f_{e \tau}\right| \simeq\left|f_{\mu \tau}\right|$.

The current constraints on the new parameters $f_{e \mu}, f_{e \tau}, f_{\mu \tau}, \mu$ in the Zee model are discussed in detail in Ref. [18. Unfortunately there are not enough experimental data to determine these parameters. Here we will only mention about the radiative decays of neutrino and charged leptons induced by Zee boson exchange [15]. In the present case the possible radiative decays are $\nu_{1(2)} \rightarrow \nu_{3}+\gamma$. Under the assumption of Eq. (55) the amplitude is given as

$$
A\left(\nu_{1} \rightarrow \nu_{3}+\gamma\right)=\frac{e}{32 \pi^{2} \bar{M}^{2}}\left(m_{1}-m_{3}\right) f_{e \mu}^{2} U_{\mu 1} U_{\mu 3} \bar{\nu}_{3} i \sigma_{\mu \nu} q^{\nu} \epsilon^{\mu} \gamma_{5} \nu_{1}
$$

where

$$
\frac{1}{\bar{M}^{2}}=\frac{\cos ^{2} \phi}{M_{1}^{2}}+\frac{\sin ^{2} \phi}{M_{2}^{2}} .
$$

Here the CP property of $\nu_{1}$ and $\nu_{3}$ is taken to be the same. The decay width and lifetime are

$$
\begin{aligned}
\Gamma\left(\nu_{1} \rightarrow \nu_{3}+\gamma\right) & =\frac{\left(m_{1}-m_{3}\right)^{3}}{8 \pi}|A|^{2} \\
& \simeq \frac{\alpha}{2}\left(\frac{f_{e \mu}^{2} U_{\mu 1} U_{\mu 3}}{32 \pi^{2} \overline{M^{2}}}\right)^{2} m_{1}^{5} \\
\tau\left(\nu_{1} \rightarrow \nu_{3}+\gamma\right) & >4 \times 10^{45}\left(\frac{2 \sqrt{2}}{U_{\mu 1}} \frac{\sqrt{2}}{U_{\mu 3}}\right)^{2}\left(\frac{3.2 \times 10^{-2}}{m_{1}(\mathrm{eV})}\right)^{5} \quad \text { years }
\end{aligned}
$$


where we have used the present limits obtained in Ref. [18]

$$
\frac{f_{e \mu}^{2}}{\overline{M^{2}}}<7 \times 10^{-4} G_{F} .
$$

The amplitude for radiative decay of charged leptons also induced by Zee boson exchange at one loop level is

$$
A(\mu \rightarrow e+\gamma)=\frac{e}{768 \pi^{2} \bar{M}^{2}} f_{\mu \tau} f_{e \tau} \bar{u}_{e}\left(p^{\prime}\right) \sigma_{\mu \nu} q^{\mu} \epsilon^{\nu}\left(1+\gamma_{5}\right) u_{\mu}(p) .
$$

For $\tau \rightarrow e \gamma$ and $\tau \rightarrow \mu \gamma$ the parameters $f_{\mu \tau} f_{e \tau}$ should be replaced with $-f_{\mu \tau} f_{e \mu}$ and $f_{e \mu} f_{e \tau}$, respectively. The branching ratio is

$$
B r(\mu \rightarrow e+\gamma)=\frac{\alpha}{3072 \pi}\left(\frac{f_{\mu \tau} f_{e \tau}}{\bar{M}^{2}}\right)^{2} \frac{1}{G_{F}^{2}}<4 \times 10^{-27}
$$

by using the upper limit (60) and Eqs.(52,53). Thus the radiative decays of neutrinos and charged leptons, induced by the Zee boson, are negligible.

In conclusion we have searched for solutions within the framework of the Zee mass matrix, that has vanishing or in general very small diagonal elements, by taking maximal mixing angle $\theta_{2} \simeq \pi / 4$ between $\nu_{\mu}$ and $\nu_{\tau}$. The solution we have found is given by Eq.(51). It corresponds to the bi-maximal solution, which requires large mixing angles for both solar- and atmospheric neutrinos. The two heaviest neutrinos $\nu_{1}$ and $\nu_{2}$, which are approximately degenerate, and the lightest neutrino $\nu_{3}$ are given by

$$
\begin{aligned}
& \nu_{1} \simeq \frac{1}{\sqrt{2}} \nu_{e}-\frac{1}{2} \nu_{\mu}+\frac{1}{2} \nu_{\tau}, \\
& \nu_{2} \simeq \frac{1}{\sqrt{2}} \nu_{e}+\frac{1}{2} \nu_{\mu}-\frac{1}{2} \nu_{\tau}, \\
& \nu_{3} \simeq \frac{1}{\sqrt{2}} \nu_{\mu}+\frac{1}{\sqrt{2}} \nu_{\tau} .
\end{aligned}
$$

The solution thus found requires a large hierarchy for the couplings of the Zee boson to leptons, in the form $f_{e \mu} \gg f_{e \tau} \gg f_{\mu \tau}$ in contrast to "natural" expectations. It is therefore desirable to impose an approximate $L_{e}-L_{\mu}-L_{\tau}$ symmetry in the Zee model in order to explain this hierarchy. The model will undergo severe tests in the future neutrino experiments.

\section{Acknowledgment}

One of the authors (M.M) expresses his thanks to the Royal Swedish Academy of Sciences (RSAS) for the support by a grant. M.T is thankful to the High Energy Group in CFIF/IST (Portugal) for their hospitality. His work is supported by the Grant-in-Aid for Science Research, Ministry of Education, Science and Culture, Japan (No.1014028, No.10640274). 


\section{REFERENCES}

[1] Super-Kamiokande Collaboration, Y. Fukuda et al. Phys.Lett. B433 (1998) 9; B436 (1998) 33; Kamiokande Collaboration, S. Hatakeyama et al., Phys.Rev.Lett. 81 (1998) 2016.

[2] MACRO collaboration, M. Ambroiso et al., Phys.Lett. B434 (1998) 451.

[3] Super-Kamiokande Collaboration, Y. Fukuda et al., Phys.Rev.Lett. 81 (1998) 1562.

[4] Super-Kamiokande Collaboration, Y. Fukuda et al., Phys.Rev.Lett. 81 (1998) 1158.

[5] GALLEX Collaboration, P. Anselmann et al., Phys. Lett. 327B (1994) 377; 388B (1996) 384; SAGE Collaboration, J. N. Abdurashitov et al., Phys.Rev.Lett. 77 (1996) 4708; Homestake Collaboration, R. Davis et al., Nucl. Phys. B38 (Proc. Suppl.) (1995) 47.

[6] Y. Suzuki, Proc. of Neutrino 96, Helsinki, June 1996, edited by K. Enqvist et al., p.237 (World Scientific, Singapore, 1997).

[7] S. G. Wojcicki, Proc. of Neutrino 96, Helsinki, June 1996, edited by K.Enqvist et al.,p.231 (World Scientific, Singapore, 1997).

[8] ICARUS Collaboration, P. Cennini et al., LNGS-94/99-I, May 1994; F. Pietropaola, invited talk at Neutrino 98, 4 - 9 June, 1998, Takayama, Japan.

[9] The CHOOZ collaboration, M. Apollonio et al., Phys.Lett. B420 (1998) 397.

[10] N. Cabibbo, Phys. Rev. Lett. 10 (1963) 531; M. Kobayashi and T. Maskawa, Prog. Theor. Phys. 49 (1973) 652.

[11] Z. Maki, M. Nakagaa and S. Sakata, Prog. Theor. Phys. 28 (1962) 870.

[12] M. Gell-Mann, P. Ramond and R. Slansky, in Supergravity, Proceedings of the Workshop, Stony Brook, New York, 1979, edited by P. van Nieuwenhuizen and D. Freedmann, North-Holland, Amsterdam, 1979, p.315; T. Yanagida, in Proceedings of the Workshop on the Unified Theories and Baryon Number in the Universe, Tsukuba, Japan, 1979, edited by O. Sawada and A. Sugamoto, KEK Report No. 79-18, Tsukuba, 1979, p.95.

[13] A. Zee, Phys. Lett. 93B, (1980) 389; 161B (1985) 141.

[14] L. Wolfenstein, Nucl. Phys. B175 (1980) 92.

[15] S. T. Petcov, Phys. Lett. 115B (1982) 401.

[16] J. Liu, Phys.Lett. B216 (1989) 367; W. Grimus and H. Neufeld, Phys. Lett. 237B (1990) 521; B. K. Pal, Phys.Rev. D44 (1991) 2261; W. Grimus and G. Nardulli, Phys.Lett. B271 (1991) 161.

[17] A. Yu. Smirnov and Z. Tao, Nucl. Phys. B426 (1994) 415.

[18] A. Yu. Smirnov and M. Tanimoto, Phys. Rev. D55 (1997) 1665.

[19] S.M. Bilenky, S.T. Petcov, Rev. Mod. Phys. 59 (1987) 671.

[20] LSND Collaboration, C. Athanassopoulos et al., Phys. Rev. Lett. 75 (1995) 2650; 77 (1996) 3082; 81 (1998) 1774; Phys. Rev. C54 (1996) 2685; J. E. Hill, Phys. Rev. Lett. 75 (1995) 2654.

[21] KARMEN Collaboration, B. Armbruster et al., hep-ex/9809007; B. Zeitnitz, invited talk at Neutrino 98, 4 - 9 June, 1998, Takayama, Japan.

[22] G. L. Fogli, E. Lisi, A. Marrone, and G. Scioscia, hep-ph/9808205.

[23] J. N. Bahcall, P. I. Krastev and A. Yu. Smirnov, Phys.Rev. D58 (1998) 6016. 
[24] Private communication with J.N. Bahcall; The large angle MSW solutions are excluded only if one fits the SK electron recoil energy spectrum, assuming the hep production cross section obtained by nuclear physics calculations. See also J. N. Bahcall and P.I. Krastev, Phys. Lett. B436 (1998) 243.

[25] S.M. Bilenky, C. Giunti, hep-ph/9802201.

[26] V. Barger, P. Pakvasa, T. J. Weiler, and K. Whisnant, Phys. Lett B437 (1998) 107; A. Baltz, A.S. Goldhaber and M. Goldhaber, Phys.Rev.Lett.58 (1998) 5730; Y. Nomura and T. Yanagida, Phys.Rev. D59 (1999) 017303; M. Jezabek and Y. Sumino, Phys.Lett. B440 (1998) 327; H.Georgi and S.L.Glashow, hep-ph/9808293; R. Mohapatra and S. Nussinov, Phys.Lett.B441 (1998) 299, hep-ph/9809415; C. Giunti, hep-ph/9810272.

[27] H. Fritzsch and Z. Xing, Phys. Lett. B372 (1996) 265; M. Fukugita, M. Tanimoto and T. Yanagida, Phys. Rev. D57 (1998) 4429; M. Tanimoto, Phys.Rev. D59 (1999) 017304.

[28] G. Giunti, hep-ph/9802201 (1998).

[29] P.F. Harrison and W. G. Scott, Phys. Lett. B333 (1994) 471; P.F. Harrison, D. H. Perkins and W. G. Scott, Phys. Lett. B349 (1995) 137; B374 (1996) 111; B396 (1997) 186.

[30] R.N. Mohapatra, hep-ph/9808284 (1998).

[31] R. Barbieri, L.J. Hall, D. Smith, A. Strumia, N. Weiner, hep-ph/9807235 (1998).

[32] N. Gaur, A. Ghosal, E. Ma and P. Roy, Phys. Rev. D58 (1998) 1301. 